\title{
Correlations of T2* Mapping with Delayed Gadolinium Enhancement in Magnetic Resonance Imaging of Chronic Myocardial Infarction
}

\author{
Ali Mohammadzadeh ${ }^{1}$, Zahra Maleki ${ }^{1}$, Ali Nahardani ${ }^{1}$ and Maryam Mohammadzadeh ${ }^{2,3,{ }^{*}}$ \\ ${ }^{1}$ Department of Radiology, Rajaie Cardiovascular and Medical Research Center, Iran University of Medical Sciences, Tehran, Iran \\ ${ }^{2}$ Department of Radiology, Amir Alam Hospital, Tehran University of Medical Sciences, Tehran, Iran \\ ${ }^{3}$ Advanced Diagnostic and Interventional Radiology Research Center (ADIR), Tehran University of Medical Sciences, Tehran, Iran \\ "Corresponding author: Maryam Mohammadzadeh, Department of Radiology, Amir Alam Hospital, Tehran University of Medical Sciences, Tehran, Iran. Tel: +98-9113386324, \\ Email:mm1361@yahoo.com
}

Received 2017 May 24; Revised 2018 June 03; Accepted 2018 June 10.

\begin{abstract}
Background: Scar tissue formation is a common phenomenon in myocardial infarctions. Contrast-enhanced cardiac magnetic resonance imaging is the modality of choice to evaluate the location and size of the scar tissue. Nevertheless, in patients with severe kidney impairment, administration of gadolinium is contraindicated.

Objectives: So the aim of this study was to substitute a safe way for myocardial infarction assessments in patients with a history of renal function impairment. We assessed the $\mathrm{T} 2^{*}$ quantitative value changes in myocardial infarction-related scar tissue and compared them with normal/remote myocardial tissue $\mathrm{T} 2 *$ values to evaluate its application in non-contrast cardiac magnetic resonance imaging viability assessments.

Patients and Methods: Twenty patients with a previous history of non ST-elevation myocardial infarction (NSTEMI) underwent cardiac magnetic resonance (CMR) examination with a 1.5T MR imaging scanner (Avanto, Siemens AG Healthcare Sector, Erlangen, Germany). The time interval between myocardial infarction occurrence and CMR assessment was at least six months. All the patients had normal kidney function. The imaging protocol consisted of three steps: the functional left-ventricular imaging; 8-echoes gradient recalled echo $\mathrm{T} 2 *$ mapping; and delayed/late gadolinium-enhancement imaging. The left-ventricle functional and T2* mapping assessments were done by CMR42 image analysis software (Circle Cardiovascular Imaging, Calgary, Canada). T2* values were calculated for 49 regions of interests (ROIs) at the infarction (14 ROIs), peri-infarction (12 ROIs), and remote/normal myocardial tissues (23 ROIs), and their means were compared statistically by the Leven's test. Finally, the receiver operator characteristics curve was calculated.

Results: $2_{2} *$ (mean \pm SD) values of the normal/remote, peri-infarction, and infarcted myocardial tissues were calculated as 29.42 $\pm 4.50,30.71 \pm 4.86$, and $35.46 \pm 3.61$, respectively. There was a significant alteration in the post-infarction scar tissue's myocardial T2* values by comparison with the normal/remote myocardium $(\mathrm{P}<0.001)$. No significant differences were noticed between the normal/remote and peri-infarction regions' $\mathrm{T} 2 *$ relaxation times $(\mathrm{P}>0.05)$. Area under the curve was $81 \%$ for patients evaluated for scars. Cutoff value of 31.70 yielded $92 \%$ sensitivity and $72 \%$ specificity.

Conclusion: $\mathrm{T}^{*}$ relaxation time can provide useful and sensitive information on scar tissue formation location, and can offer a greater sensitivity into the non-contrast CMR imaging viability assessments besides other relaxation phenomena, especially the longitudinal relaxation time.
\end{abstract}

Keywords: Cardiac Magnetic Resonance Imaging, T2* Mapping, Delayed Gadolinium-Enhancement Imaging, Myocardial Infarction

\section{Background}

Myocardial ischemia is defined as the insufficiency of blood supply which results in inadequate oxygenation to the myocardial tissue (1). Whenever there is a reduction in myocardial blood flow, myocardial tissue adapts itself to the newly introduced oxygenation level (2). When blood flow reduces beyond tolerance of the myocardial tissue, necrosis takes place, a condition that is called myocardial infarction (MI) $(1,3)$. In this manner, myocardial functionality is reduced (4). The ischemic myocardium can be divided into two categories: the stunned myocardium; and the hibernating myocardium (5). Stunned myocardium is the predominant consequence of acute myocardial ischemia with myocardial dysfunction but responds positively to reperfusion in short term. On the other hand, hibernating myocardium, which primarily occurs because of long-term hypo-perfusion, or multiple acute ischemia of 
the myocardial tissue may respond either positively or negatively to revascularization (6). While underlying mechanisms of stunning and hibernating myocardial regulatory processes are not completely understood, it is hypothesized that both conditions benefit from downregulation of myocyte metabolism (7). There is a strong correlation between increasing hypoxia stress duration on the myocardium and its damage reversibility. Therefore, diagnosis of viable myocardium is crucial in treatment planning of patients experiencing chronic MI (6).

There are different modalities to evaluate the viability status of the myocardium. To date, stress-radionuclide imaging, stress-echocardiography, and magnetic resonance imaging techniques have been introduced as the main approaches for such evaluations $(8,9)$. Stressradionuclide imaging employs the emission of gamma ionizing radiation radiopharmaceuticals in its setting, thus it has hazardous effects on biological tissues $(10,11)$. Stress-radionuclide imaging cannot acquire the data of the myocardial functionality and can only evaluate myocardial viability by its perfusion patterns and late enhancements. Stress-radionuclide scan also cannot evaluate the subendocardial infarction and yields a low sensitivity in those regions (11, 12). Recent advances have led to the emergence of positron emission tomography (PET). The fluorine-18 fluorodeoxyglucose (18F-FDG) PET has a higher sensitivity and specificity in myocardial viability assessments by comparison with Thallium 201 SPECT imaging. In addition, PET benefits from a variety of radiotracers, such as Rb-82, $\mathrm{N}-13$ ammonia, and $\mathrm{O}-15 \mathrm{H}_{2} \mathrm{O}$. Studies have demonstrated that myocardial perfusion assessments by these radiotracers would result in a higher diagnostic accuracy in coronary artery disease (CAD) evaluations in comparison to other radionuclide imaging modalities and methods. This issue has great importance in the balanced reduction of myocardial perfusion due to three-vessel or mainstem CAD (13). Stress-echocardiography can evaluate the myocardial viability status just by functional analysis but is unable to assess the structural and histological changes on its own $(14,15)$. Contrary to this, cardiac magnetic resonance (CMR) imaging can evaluate myocardial perfusion, histological changes, and functionality. Thus, it is the modality of choice for viability assessments $(6,16)$.

In CMR imaging viability assessments, there is a need for gadolinium-based contrast material injection to evaluate the myocardial rest-perfusion, early gadolinium enhancement (EGE) and late gadolinium enhancement (LGE) in order to rule out the hypo-perfusion, no-reflow zone, and scar tissue formation (16). Unfortunately, in patients with renal failure and hypersensitivity to gadoliniumbased contrast agents, the injection of gadolinium is contraindicated and there is a need for introduction of a mag- netic resonance criterion to make it possible to evaluate myocardial viability status (17). Previous studies have proved the development of an inflammatory process after acute MI, while there would be tissue alteration and scar formation in chronic ones $(18,19)$. As such, the synced inflammation in acute MI and tissue alteration in chronic MI would result in different magnetic properties and thus in different $\mathrm{T} 1 / \mathrm{T} 2 / \mathrm{T} 2^{*}$ relaxation times (20). In recent years, numerous studies have evaluated the role of cardiac $\mathrm{T} 1$ and T2 mapping in MI or viability assessments. Studies have showed a high sensitivity of T2 mapping techniques in edema formation in acute MI, and T1 mapping techniques in fibrosis evaluations. In these conditions, both the native $\mathrm{T} 1$ and $\mathrm{T} 2$ relaxation times are prolonged (21). Although myocardial T1 and T2 mapping techniques are becoming increasingly popular worldwide, there should be more investigations in this field. $\mathrm{T}^{*}$ mapping is also a popular method for post-revascularization hemorrhage imaging. By this method, clinicians can determine the extent of ischemic area at risk. This technique is usually performed post reperfusion procedures. These studies have hypothesized that the post-reperfusion hemorrhage extent might be a good representative of the microvascular obstruction (MVO). MVO is considered as nonviable myocardial volume in CMR assessments (22). In fact, post revascularization $\mathrm{T}^{*}$ mapping would reveal MVO and micro-hemorrhage as a post MI adverse effect. In this study, we hypothesized that the pre-revascularization $\mathrm{T}^{*}$ mapping could be a strong indicator of MI extent. In contrast with previous studies (O Regan et al., Komar et al., and Hamirani et al.), we selected our patients at prerevascularization stage of MI therapy to search for the possibility of T2* relaxometry potential in myocardial scar tissue detection. Although there are numerous literatures about the native cardiac $\mathrm{T} 1$ and $\mathrm{T} 2$ values changes in different cardiovascular diseases, few studies have been conducted to evaluate myocardial T2* changes in MI. Therefore, in our research, we decided to evaluate native myocardial $\mathrm{T}^{*}$ value changes in patients with $\mathrm{MI}$ and assess the technique for myocardial fibrosis detection in prerevascularization stages.

\section{Objectives}

The aim of this study was to assess $\mathrm{T} 2^{*}$ mapping as an alternative to gadolinium enhanced sequence for delineation of infarcted zone in patients suffering from chronic myocardial infarction who specifically have restrictions for gadolinium injection. 


\section{Patients and Methods}

\subsection{Study Population}

In this study, 20 patients with confirmed non STelevation myocardial infarction (NSTEMI) electrocardiographic (ECG) changes (mean age of 60, 13 males and 7 females in the age range of 26 - 79) underwent CMR examinations for viability assessments by $1.5 \mathrm{~T}$ cardiac MR imaging (Avanto, Siemens AG Healthcare Sector, Erlangen, Germany). The time interval between MI occurrence and CMR assessment was at least six months. The patients' demographic information is provided in Table 1 . All the national and international ethical human research rules were supervised by the institution's ethical research committee and followed completely by the researchers.

\subsection{CMR Examination}

All CMR examinations were performed using a 1.5T MR system (Avanto, Siemens, Erlangen, Germany). The protocol was identical for all patients. A16-channel phased-array body RF coil was employed for signal reception in all patients. All the sequences were ECG-gated either retrospectively or prospectively.

\subsubsection{Cine Imaging}

True-fast imaging with steady state precession (TrueFISP) 2D Breath-hold cine images was obtained retrospectively in the two-chamber, four-chamber, three-chamber, left ventricular output tract, and short-axis views in all the patients to assess the left ventricular wall motion kinesis qualitatively and to measure its indices quantitatively. The sequence parameters are given in Table 2 .

\subsection{2. $T 2 *$ Mapping}

The cardiac $\mathrm{T}_{2} *$ relaxometry data were acquired by a breath-hold, bright-blood, gradient-recalled echo pulse sequence which could obtain eight signals with different echo-times (TE) in each repetition-time (TR). The patients' hearts are imaged in the three basal, mid-ventricular and apical short-axis, two-chamber and four-chamber views by the given parameters in Table 2 .

\subsubsection{LGE Imaging}

The LGE images were acquired $10 \mathrm{~min}$ after the gadolinium-based contrast agent administration by the True-FISP Phase-Sensitive Inversion-Recovery (True-FISP PSIR) pulse sequence in the two-chamber, four-chamber, and short-axis views. Gadoteric acid under the brand name of Dotarem (Guerbet, France) was administered to all patients. The contrast media injection dose and flow rate were $0.2 \mathrm{mmol} / \mathrm{kg}$ and $4 \mathrm{~mL} / \mathrm{s}$, respectively. All the LGE

\begin{tabular}{|c|c|}
\hline Variables & Value \\
\hline \multicolumn{2}{|l|}{ Gender } \\
\hline Male & 13 \\
\hline Female & 7 \\
\hline \multicolumn{2}{|l|}{ Age, $y$} \\
\hline $20-29$ & 1 \\
\hline $30-49$ & 3 \\
\hline $50-64$ & 9 \\
\hline$>65$ & 7 \\
\hline \multicolumn{2}{|l|}{ Hyperlipidemia } \\
\hline Yes & 9 \\
\hline No & 11 \\
\hline \multicolumn{2}{|l|}{ Smoking } \\
\hline Yes & 8 \\
\hline No & 12 \\
\hline \multicolumn{2}{|l|}{ Diabetes } \\
\hline Yes & 12 \\
\hline No & 8 \\
\hline \multicolumn{2}{|l|}{ Hypertension } \\
\hline Yes & 11 \\
\hline No & 9 \\
\hline \multicolumn{2}{|l|}{ ECG } \\
\hline NSTEMI & 20 \\
\hline STEMI & 0 \\
\hline \multicolumn{2}{|c|}{ Myocardial infarction } \\
\hline Acute & 0 \\
\hline Chronic & 20 \\
\hline \multicolumn{2}{|c|}{ Planned for CABG } \\
\hline Yes & 7 \\
\hline No & 13 \\
\hline
\end{tabular}

Abbreviations: CABG, coronary artery bypass grafting; ECG, electrocardiographic; NSTEMI, non ST-elevation myocardial infarction; STEMI, ST-elevation myocardial infarction.

images were acquired in swapping phase direction again to eliminate any artifactual false positives from the final analysis (Table 2).

\subsubsection{Pre-/Post-Contrast T1W Imaging}

Pre-/Post-contrast T1w imaging is always done by the MRI's working horse pulse sequence, the Turbo-Spin Echo. Its applied parameters are given in Table 2. The imaging plane of choice in the cardiac T1w imaging is transverse and it is always gated during the systolic activity of the 


\begin{tabular}{|c|c|c|c|}
\hline Sequence & GRE- 8 echoes & True-FISP PSIR & Turbo-spin echo \\
\hline TR/TE, ms & $200 /(2.59,4.82,7.05,9.28,11.51,13.74,15.97,18.20)$ & $800 / 1.02$ & $281 / 13$ \\
\hline TI, ms & - & 300 & - \\
\hline Flip angle & 20 & 40 & 160 \\
\hline Pixel size, mm & $2.08 \times 2.08$ & $1.06 \times 1.06$ & $1.6 \times 0.8$ \\
\hline $\mathbf{B W}, \mathbf{H z} / \mathbf{P x}$ & 815 & 1420 & 109 \\
\hline Averages & 1 & 1 & 4 \\
\hline ECG-Gating & Prospective-systole & Prospective-diastole & Prospective-systole \\
\hline Application & $\mathrm{T} 2 *$ mapping & LGE imaging & Hyperemia \\
\hline
\end{tabular}

Abbreviations: BW, bandwidth; ECG, electrocardiography; FISP, fast imaging with steady-state precession; FOV, field-of- view; GRE, gradient echo; GRE-8 Echoes, gradient recalled echo with 8 TEs; LGE, late gadolinium enhancement; PSIR, phase-sensitive inversion recovery; TE, echo-times; TI, inversion time; TR, repetition-time.

heart.

\subsection{Image Analysis}

The acquired left-ventricular functional images were analyzed both qualitatively and quantitatively by two, fiveyear experienced, cardiothoracic radiologists using $\mathrm{CMR}^{42}$ (Circle Cardiovascular Imaging, Calgary, Canada) cardiac MRI analysis software. The T2* relaxation times were quantified by an experienced clinical MRI physicist in the normal remote, peri-infarction myocardium, and LGE regions by the same software. All analysis was done in a singleblind manner. Mean T2* values in the proven LGE, periand remote myocardial regions were compared statistically by the SPSS v.22 software (IBM Corp. Released 2013. IBM SPSS Statistics for Windows, Version 22.0. Armonk, NY: IBM Corp.) to assess any significant differences between these regions. Finally, receiver operator characteristics (ROC) curves were drawn and Youdin index and area under curve (AUC) were calculated.

\section{Results}

Out of the 20 sampled patients, 13 patients were diagnosed with no myocardial fibrosis and seven patients with myocardial scars on the LGE images; out of these seven positive patients with delayed gadolinium enhancement (DGE), four patients were diagnosed with the left ascending artery (LAD) territory DGE (Figure 1) and three with the right coronary artery (RCA) territory scar tissue formations (Figure 2). In total, 49 regions of interest (ROIs) were drawn on the GRE-8echoes images: 14 ROIs on the infarcted myocardium; 23 ROIs on the normal/remote myocardium; and 12 ROIs on peri-infarction myocardial tissues. All the DGE images were correlated with the cinefunctional short-axis views to eliminate any artifactual dis- crimination between the results. The mean $\pm \mathrm{SDT} 2 *$ values of the normal/remote myocardium, peri-infarction myocardium, and delayed enhancement region were calculated as $29.42 \pm 4.50,30.71 \pm 4.86$, and $35.46 \pm 3.61$ milliseconds, respectively, as is shown in Table 3.

Table 3. Normal/Remote, Peri-Infarction, and Infarcted Myocardial Mean \pm SD T2* Values

\begin{tabular}{lcc}
\hline Myocardium & No. & Mean \pm SD \\
\hline Delayed enhanced myocardium & 14 & $35.46 \pm 3.62$ \\
Peri-infarction myocardium & 12 & $30.72 \pm 4.87$ \\
Remote/normal myocardium & 23 & $29.42 \pm 4.51$ \\
\hline
\end{tabular}

Abbreviations: SD, standard deviation.

Following the mean $\mathrm{T}^{*}$ calculations, two mean comparisons Leven's tests (within the confidence interval [CI] of 0.95 ) were performed to analyze the mean differences significance between the results. The performed t-tests revealed a significant difference between the infarcted and normal/remote myocardial $\mathrm{T} 2 *$ values $(\mathrm{P}<0.001)$, and a non-significant difference between the normal/remote and peri-infarction $\mathrm{T}^{*}$ measurements $(\mathrm{P}>0.05)$. The ROC curve was drawn and AUC was $81 \%$ in the CI of $95 \%$. The analysis cutoff value of 31.70 yielded $92 \%$ sensitivity and $72 \%$ specificity in the final results (Figure 3 ).

\section{Discussion}

CMR imaging plays a pivotal role in the diagnosis, risk stratification and management of chronic MI patients. This modality of imaging brings useful information regarding left ventricular function, remodeling, edema, the infarcted myocardium substituting scar tissue size, and patients' treatment strategies. In the chronic stage of MI, 

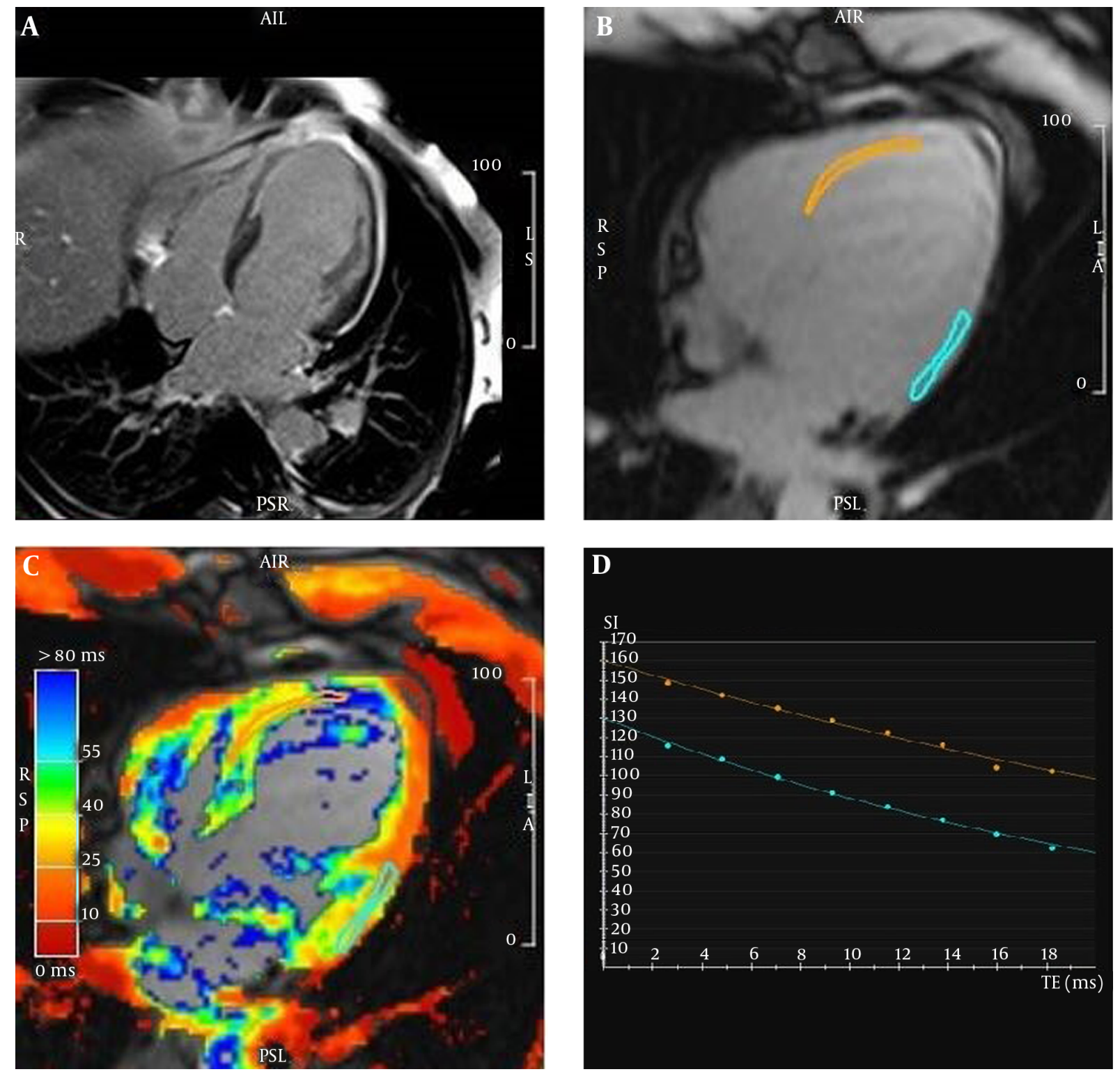

Figure 1. A patient with myocardial infarction. A and B, Depict chronic myocardial infarctions in the left ascending artery (LAD) and partially left circumflex artery (LCX) territories. B and C, As can be noticed, two orange and blue regions of interest (ROIs) were drawn on the fibrous and normal tissues, respectively. D, The ROIs' T2* signal decay curves show a significant difference between the scar and remote healthy myocardial T2* relaxation values. The fibrous (orange) and normal remote myocardial (blue) T2*relaxation times equal to 41 and $27.7 \mathrm{~ms}$, respectively.

LGE imaging and its functional correlations are of special importance among all the others. Unfortunately, the administration of gadolinium is contraindicated in patients with acute or chronic severe renal impairments (with a glomerular filtration rate of less than $30 \mathrm{ml}$ per min per 1.73 square meters) due to the risk of the nephrogenic systemic fibrosis $(17,23)$. Thus, an alternative imaging method to depict scar tissue formation without use of the administration of gadolinium-based contrast agents could be of value. Several studies have evaluated the role of precontrast T1 and T2 mapping techniques in the diagnosis of MI. In 2014, Hamlin et al. reported an increase in the postinfarction scar tissue pre-contrast T1 values by comparison with the normal/remote regions (21). Another study conducted by Caudron et al. in 2013 revealed the same result (24). The two afore-mentioned studies are just two examples of tens of reports that have evaluated the role of T1 and T2 mapping techniques in the diagnosis of MI. 

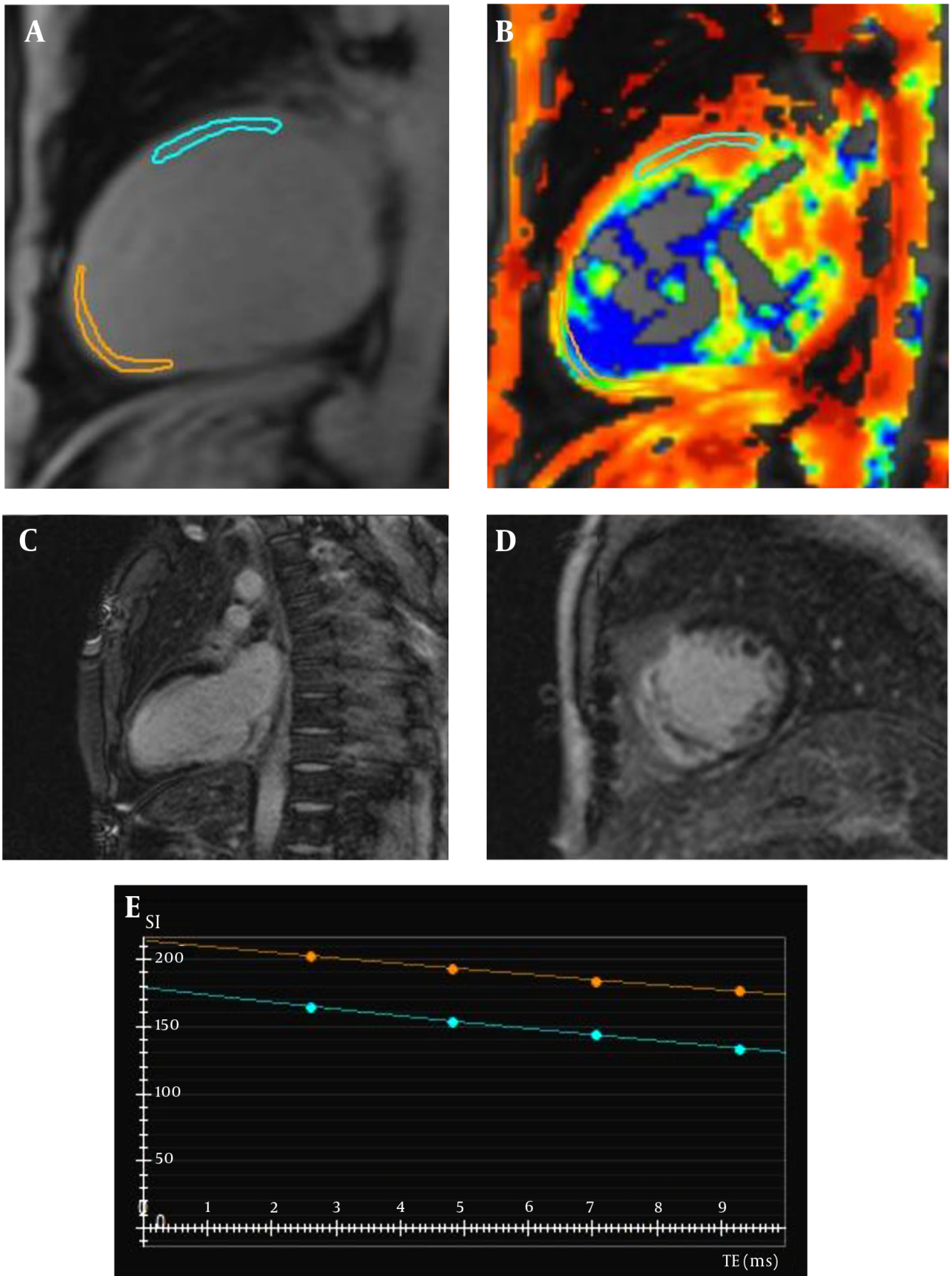

Figure 2. A 50-year-old male patient with myocardial infarction. A and B, Depict the myocardial T2* mapping. There are two regions of interest drawn on the infarcted myocardium (orange) and the remote normal area (blue). C and D, Two-chamber and short-axis views, show delayed gadolinium transmural enhancement in the left ascending artery (LAD) and right coronary artery (RCA) territories. E, Depict the different T2* decay curves in the infarcted (orange, $47.73 \mathrm{~ms}$ ) and remote normal myocardium (blue, 32.03 $\mathrm{ms})$. 


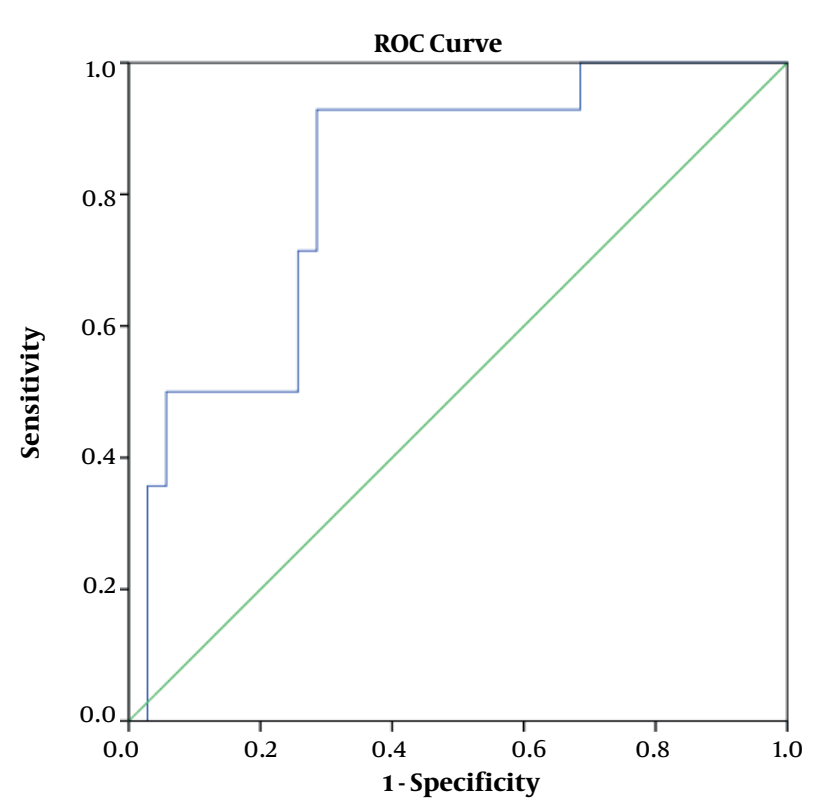

Figure 3. The receiver operator characteristic(ROC) curve for T2* values with $95 \%$ confidence interval $(\mathrm{CI})$ to categorize the scar tissue from normal or peri-infarct tissue. The area under curve (AUC) was 81.6 ( $\mathrm{P}$ value $<0.001$ ) for T2* to differentiate between scar and non-scar tissues.

However, few studies have attempted to evaluate the $\mathrm{T} 2 *$ quantitative value changes in this condition, although it was a more sensitive parameter to tissue alterations. The $\mathrm{T} 2 *$ relaxation time is composed of two elements, the T2 and $\mathrm{T} 2 *$. On the other hand, the $\mathrm{T} 2 *$ relaxation time is affected by the spin-spin interaction effect and local magnetic field susceptibility changes. As a result, the T2* relaxation time is a more sensitive measure to evaluate the structural and pathological changes in tissues. In this study, we hypothesized that there would be significant rise in the post-infarction fibrotic tissue's T2* relaxation times in comparison with the normal/remote myocardium. Although the sample population of this study was small, our alternative hypothesis was proved significantly $(\mathrm{P}<0.001)$, and we noticed a significant increase in the chronically infarcted myocardium compared with the normal/remote myocardial tissue. We found that cutoff value of 31.70 is sensitive in differentiating scar tissue from non-scar tissue. We believe that the $\mathrm{T} 2 *$ relaxation time measurement would be a sensitive tool for tissue composition changes, but not an specific tool to tell us what exactly the composition would be, while the inflammatory or iron-deposition process could change these values. Also, the MVO and its underlying different magnetic susceptibility may decrease the study's specificity to a great extent, while we did not differentiate between the scar tissue's composition with or without the microvascular obstruction. On the other hand, if we could completely rule out confounding factors of any $\mathrm{T}^{*}$ changes clinically, we could correlate the $\mathrm{T} 2 *$ relaxation time changes with a specific tissue alteration in each myocardial disease. Undoubtedly, evaluation of the $\mathrm{T} 2 *$ relaxation times by $\mathrm{T} 1$ and $\mathrm{T} 2$ values could increase the non-contrast CMR's sensitivity in MI evaluations. Every cardiac sequence might suffer from several common limitations and pitfalls that decrease the image quality, including patients' lack of cooperation, cardiac arrhythmia, and claustrophobia. Like all other prospective researches, our study suffered from limited sample size and number of the analyzing ROIs, which could result in a lower reliability of the ultimate results. In addition, we did not measure the MVO T2* values at the pre-revascularization stage due the probable concurrence of scar tissue and MVO formation in the same coronary site. Furthermore, we highly recommend further research be conducted to investigate for any statistical correlations between the pre- and the postrevascularization stages.

In conclusion, CMR plays a vital role in the management of chronic MI. Although the viability assessments in cardiac MRI requires an injection of gadolinium-based contrast agents for delayed enhancement and scar tissue imaging, their administration is fully contraindicated in patients with severe renal failure. Through CMR technology developments in recent years, we have arrived to the point to address this issue. CMR relaxometry is a promising technique to evaluate myocardial tissue changes in non-contrast CMR by measuring $\mathrm{T} 1, \mathrm{~T} 2$, and $\mathrm{T} 2$ * relaxation times changes in any myocardial disease. In this study, in the event of any concomitant conditions or diseases being ruled out correctly by the reporting and performing clinicians, We proved that $\mathrm{T} 2 *$ relaxation time could be a sensitive measurement to diagnose myocardial scar tissue in patients with history of myocardial infarctions.

\section{Footnotes}

Authors' Contributions: All authors have contributed in this study in terms of study design, image interpretation, statistical analysis, scientific writing and revisions.

Ethical Approval: The study was approved by Research Committee of Iran University of Medical Sciences.

Financial Disclosure: None declared.

Funding/Support: The study was funded by Rajaie Cardiovascular Medical and Research Center.

\section{References}

1. Cohn PF, Fox KM, Daly C. Silent myocardial ischemia. Circulation. 2003;108(10):1263-77. doi: 10.1161/01.CIR.0000088001.59265.EE. [PubMed: 12963683]. 
2. Patel DJ, Knight CJ, Holdright DR, Mulcahy D, Clarke D, Wright C, et al. Pathophysiology of transient myocardial ischemia in acute coronary syndromes. Characterization by continuous ST-segment monitoring. Circulation. 1997;95(5):1185-92. [PubMed: 9054848].

3. Mehta LS, Beckie TM, DeVon HA, Grines CL, Krumholz HM, Johnson $\mathrm{MN}$, et al. Acute Myocardial Infarction in Women: A Scientific Statement From the American Heart Association. Circulation. 2016;133(9):916-47. doi: 10.1161/CIR.0000000000000351. [PubMed: 26811316].

4. Panidis IP, Morganroth J. Two dimensional echocardiography in acute myocardial infarction: Clinical applications. In: Morganroth J, Moore EN, editors. Interventions in the acute phase of myocardial infarction. 41. US: Boston, MA: Springer; 1984. p. 88-102. doi: 10.1007/978-14613-3819-2_8.

5. Conti CR. The stunned and hibernating myocardium: a brief review. Clin Cardiol. 1991;14(9):708-12. doi: 10.1002/clc.4960140903. [PubMed: 1742904].

6. Shan K, Constantine G, Sivananthan M, Flamm SD. Role of cardiac magnetic resonance imaging in the assessment of myocardial viability. Circulation. 2004;109(11):1328-34. doi: 10.1161/01.CIR.0000120294.67948.E3. [PubMed:15037539].

7. Sawyer DB, Loscalzo J. Myocardial hibernation: restorative or preterminal sleep? Circulation. 2002;105(13):1517-9. [PubMed: 11927510].

8. Camici PG, Prasad SK, Rimoldi OE. Stunning, hibernation, and assessment of myocardial viability. Circulation. 2008;117(1):103-14. doi: 10.1161/CIRCULATIONAHA.107.702993. [PubMed: 18172050].

9. Wu KC, Lima JA. Noninvasive imaging of myocardial viability: current techniques and future developments. Circ Res. 2003;93(12):114658. doi: 10.1161/01.RES.0000103863.40055.E8. [PubMed: 14670830].

10. Einstein AJ, Moser KW, Thompson RC, Cerqueira MD, Henzlova MJ. Radiation dose to patients from cardiac diagnostic imaging. Circulation. 2007;116(11):1290-305. doi: 10.1161/CIRCULATIONAHA.107.688101. [PubMed: 17846343].

11. Burrell S, MacDonald A. Artifacts and pitfalls in myocardial perfusion imaging. J Nucl Med Technol. 2006;34(4):193-211. quiz 212-4. [PubMed: 17146108].

12. Mahrholdt H, Klem I, Sechtem U. Cardiovascular MRI for detection of myocardial viability and ischaemia. Heart. 2007;93(1):122-9. doi: 10.1136/hrt.2005.071290. [PubMed: 17170353]. [PubMed Central: PMC1861365].

13. Sarikaya I. Cardiac applications of PET. Nucl Med Commun. 2015;36(10):971-85. doi: 10.1097/MNM.0000000000000346.
[PubMed: 26035516].

14. Zaglavara T, Haaverstad R, Cumberledge B, Irvine T, Karvounis H, Parharidis G, et al. Dobutamine stress echocardiography for the detection of myocardial viability in patients with left ventricular dysfunction taking beta blockers: accuracy and optimal dose. Heart 2002;87(4):329-35. doi: 10.1136/heart.87.4.329. [PubMed: 11907003]. [PubMed Central: PMC1767072].

15. Marzullo P, Parodi O, Sambuceti G, Reisenhofer B, Gimelli A, Giorgetti A, et al. Myocardial viability: nuclear medicine versus stress echocardiography. Echocardiography. 1995;12(3):291-302. doi: 10.1111/j.15408175.1995.tb00552.x. [PubMed: 10150475].

16. Perazzolo Marra M, Lima JA, Iliceto S. MRI in acute myocardial in farction. Eur Heart J. 2011;32(3):284-93. doi: 10.1093/eurheartj/ehq409. [PubMed: 21112897].

17. Perazella MA. Gadolinium-contrast toxicity in patients with kidney disease: nephrotoxicity and nephrogenic systemic fibrosis. Curr Drug Saf. 2008;3(1):67-75. [PubMed: 18690983].

18. Frangogiannis N. The inflammatory response in myocardial infarction. Cardiovasc Res. 2002;53(1):31-47. doi: 10.1016/s00086363(01)00434-5.

19. Ertl G, Frantz S. Healing after myocardial infarction. Cardiovasc Res. 2005;66(1):22-32. doi: 10.1016/j.cardiores.2005.01.011. [PubMed: 15769445].

20. Kim PK, Hong YJ, Im DJ, Suh YJ, Park CH, Kim JY, et al. Myocardial T1 and T2 mapping: Techniques and clinical applications. Korean J Radiol. 2017;18(1):113-31. doi: 10.3348/kjr.2017.18.1.113. [PubMed: 28096723] [PubMed Central: PMC5240500].

21. Hamlin SA, Henry TS, Little BP, Lerakis S, Stillman AE. Mapping the future of cardiac MR imaging: case-based review of T1 and T2 mapping techniques. Radiographics. 2014;34(6):1594-611. doi 10.1148/rg.346140030. [PubMed: 25310419].

22. Kali A, Tang RL, Kumar A, Min JK, Dharmakumar R. Detection of acute reperfusion myocardial hemorrhage with cardiac MR imaging: T2 versus T2. Radiology. 2013;269(2):387-95. doi: 10.1148/radiol.13122397. [PubMed: 23847253]. [PubMed Central: PMC3807083].

23. Reiter T, Ritter O, Prince MR, Nordbeck P, Wanner C, Nagel E, et al. Minimizing risk of nephrogenic systemic fibrosis in cardiovascular mag netic resonance. J Cardiovasc Magn Reson. 2012;14:31. doi: 10.1186/1532429X-14-31. [PubMed: 22607376]. [PubMed Central: PMC3409035].

24. Caudron J, Lefebvre V, Dubourg B, Fares J, Dacher JN. T1 and T2 mapping for the detection of myocardial edema in acute myocardial infarction by cardiac magnetic resonance. Radiological Society of North America 2013 Scientific Assembly and Annual Meeting. 2013. 